\title{
Cellular-D2D resource reuse algorithms based on proportional fairness
}

Wu Zheng ${ }^{1 *} \mathbb{D}$, Jing $\mathrm{Hu}^{2}$, Chen $\mathrm{Liu}^{2}$ and Youhua $\mathrm{Fu}^{2}$

\begin{abstract}
Device-to-device (D2D) communication can improve coverage, spectrum efficiency, and energy efficiency based on the current cellular network architecture. The fair scheduling for D2D communication in the orthogonal frequency division multiple access-based cellular network is studied in this paper. Based on the proportional fairness criteria, the optimization objective is formulated to maximize the sum of the relative achievable rate of both cellular users (CUs) and D2D pairs. It is a hybrid optimization problem since it involves in both the resource reuse and the transmit power control. A practical proportional fairness scheduling (PFS) algorithm is proposed. Firstly, the admissible area is decided according to the transmit power limitation of terminals and the quality of service (QoS) requirement. Next, we seek the optimal transmit power combination for the CU and D2D pair in the admissible area and it is demonstrated that the optimal value can be selected from several points on the border of the admissible area. Lastly, when multiple subchannels can be employed for scheduling, based on the generated bipartite graph, Hungarian algorithm is adopted to realize maximum matching, i.e., select reusable CUs and D2D pairs to maximize the sum of the relative achievable rates. The simulation results show that PFS algorithm can guarantee the excellent performance of both throughput and fairness.
\end{abstract}

Keywords: D2D, Resource reuse, Proportional fairness, Relative achievable rate

\section{Introduction}

Device-to-device (D2D) communication refers to the direct communication between proximity terminals without the involvement of the base station. Due to the frequency reuse employed by short-distance transmission, the network capacity is greatly increased and the traffic load across the base station and core network is reduced, which leads to lower deployment costs and operating costs for mobile network operators $[1,2]$. As one of the key techniques of 4th and 5th generation mobile network architecture evolution, D2D communication can improve system throughput and energy efficiency, especially for emergency communication and the typical mobile Internet applications, e.g., social networks. When the D2D user reuses the resources allocated to the cellular user $(\mathrm{CU})$, the interference yields and thus the resource scheduling scheme needs to be implemented to protect CUs and improve overall performance.

Recently, several interference control and resource scheduling schemes are developed for D2D communication.

\footnotetext{
* Correspondence: zhengwu@jit.edu.cn

${ }^{1}$ Jinling Institute of Technology, Nanjing 211169, China

Full list of author information is available at the end of the article
}

Although interference control for D2D communication appears to the natural extension of that for traditional cellular communication, the key difference lies in the predefined quality of service (QoS) guarantee required for CUs. Without enforcing the QoS constraints in the throughput maximization, the D2D user is allocated to more resources for its short-distance transmission and better transmission conditions, which causes the unfairness for CUs [3]. Therefore, the target of interference control is to maximize the throughput on the condition of satisfying the signal to interference and noise ratio (SINR) requirement $[4,5]$. Based on QoS guarantee and maximum transmit power limitation, the maximum sum rate criterion is employed to set the optimal power and further decide whether the D2D users and the CUs can reuse the same resources or not [5]. Three D2D working modes, classified as cellular, orthogonal, and non-orthogonal resource reuse, are defined, and the resource allocation, power control, and mode selection are utilized to maximize throughput [6, 7]. In detail, given the conditions of no imperfect channel state information, power, and outage probability limitation, the maximization of ergodic sum rate is obtained [8]. Both centralized and 
distributed power control algorithms are investigated to limit the interference introduced by D2D communication and thus to guarantee the coverage probability of CUs, and also in the same recourse, D2D links are reused as much as possible [9]. The aforementioned papers just devote to maximize the throughput as the target of resource scheduling, which usually bring the results that some users occupy the resources for a long time and others lose the opportunities to be served, and thus the fairness among the users is a key performance index in order to improve the degree of satisfaction of each user. For the general multi-user communication, there are various fairness metrics, e.g., proportional fairness [10, 11], max-min fairness [12], and channel access fairness [13]. The concept of proportional fairness is introduced and applied to schedule D2D users in the multi-cell scenario in [14], where a stepwise resource allocation mode is employed, i.e., a CU is first selected based on the proportional fairness criterion, and then a D2D pair is randomly selected to reuse the resource. In [15], fractional frequency reuse is adopted to depress the interference between CUs and D2D users, and the proportional fairness-based resource allocation is implemented by optimizing the transmit power of D2D pair under the premise of the fixed transmit power of the CU. In [16], only D2D communications are considered and there is no resource reuse between the CUs and D2D pairs. The resources are divided into the dedicated resource area and the contention resource area. A two-step method is employed for scheduling D2D pairs: classify the D2D pairs into the dedicated resource area and contention resource area based on the computed SINR threshold and use the proportional fairness criterion to perform resource assignment in the dedicated resource area and in the contention resource area implement the resource reuse among multiple D2D pairs to achieve reuse gain. The theoretical analysis has demonstrated that the proportional fairness is the optimal tradeoff between throughput and fairness, which has the explanation from game theory [17]. The current research on the proportional fairness scheduling of the CUs and D2D pairs is usually simplified by some additional limitations, e.g., just the proportional fairness for CUs or the fixed transmit power for the CU. Inspired by these works, we present a framework of resource allocation for D2D communications underlaying cellular networks to maximize the sum of relative achievable rate of the CUs and D2D pairs while guaranteeing the QoS requirement of both CUs and D2D pairs. The framework includes three parts. First, the proportional fairness criterion is applied to achieve the sum of relative achievable rate of the CU and D2D pair. Then, the optimal power control scheme is investigated for each D2D pair and its possible $\mathrm{CU}$ partner to maximize the sum of relative achievable rate. Lastly, a maximum weight bipartite matching-based scheme is designed to determine a specific CU partner for each D2D pair when multiple subchannels are employed.

The remainder of this paper is organized into the following sections. The scenario and the system model are introduced in Section 2. The optimization problem formulation of resource reuse for one CU vs. one D2D user based on the proportional fairness criterion is proposed in Section 3, and also the procedures of the optimal solution, with the name of proportional fairness scheduling (PFS) algorithm, are derived. In Section 4, the scheme for the resource reuse between multiple CUs and multiple D2D users is described when there are multiple subchannels. The system simulation for PFS algorithm is realized and the results are analyzed in Section 5. And the conclusion remarks are summarized in Section 6.

\section{The scenario and system model}

We consider a single cell in an orthogonal frequency division multiple access (OFDMA)-based wireless cellular network where the base station (BS) is at the center and there are $N$ active CUs and $M$ pairs of D2D users within its coverage, and the sets of active CUs and D2D pairs are denoted by $\mathbb{C}=\left\{C_{1}, C_{2}, \cdots, C_{i}\right.$, $\left.\cdots, C_{N}\right\}$ and $\mathbb{D}=\left\{D_{1}, D_{2}, \cdots, D_{j}, \cdots, D_{M}\right\}$, respectively. In order to improve the spectrum efficiency of cellular systems, D2D pairs can be scheduled to reuse the uplink (UL) resources of CUs. This concept is motivated by the recognition that UL spectrum is often under-utilized as opposed to the downlink (DL) spectrum because of the asymmetric DL-UL traffic characteristics. In addition, since the UL spectrum sharing only affects the interference at the BS, the resulting interference can be better handled by the more powerful BS. D2D communication, with the name of sidelink communication, is defined in the 3rd Generation Partnership Project (3GPP) specification [18], and the mobile station (MS) supporting D2D communication can operate in two modes for resource allocation.

Mode 1: The MS requests transmission resources from the BS. The BS schedules transmission resources for transmission of D2D control information and data - Mode 2: A MS on its own selects resources from resource pools, which are denoted by the base station beforehand, and performs transport format selection to transmit D2D control information and data.

Mode 1, employed in our paper, is the fully centralized method, where the BS should know all the channel state information, and the MS needs to report the channel state information to the BS. In Mode 1, some extra overhead on control information, including the channel state 
information reporting and resource request message, has to be paid; the fully centralized scheduling method implemented by the BS can better match CUs and D2D pairs; and the appropriate resource allocation and power control can improve the transmit efficiency for the traffic data. When the mobile terminals have the feature of the static or low mobility, the channel state information can keep unchanged within a long time interval and thus the overhead on control information can be alleviated. In addition, we assume that there are $K$ orthogonal subchannels which are scheduled for CUs and D2D pairs based on the channel state information and QoS requirement.

We assume one of the $K$ subchannel sharings for one $\mathrm{CU}$ and one D2D pair denoted by CU $i$ and D2D pair $j$ as shown in Fig. 1, where $g_{i, B}$ and $g_{j}$ denote the channel gain from $\mathrm{CU} i$ to the $\mathrm{BS}$ and the channel gain between D2D pair $j$, respectively. For the interference channel, the channel gain from the transmitter of D2D pair $j$ to the $\mathrm{BS}$ and the channel gain from $\mathrm{CU} i$ to the receiver of D2D pair $j$ are expressed by $h_{j, B}$ and $h_{i, j}$, respectively. Let $p_{i}$ and $p_{j}$ denote the transmit power of $\mathrm{CU} i$ and D2D pair $j$, respectively. Since the subchannel is reused by $\mathrm{CU} i$ and D2D pair $j$, they have the same additive Gaussian white noise power, denoted by $\sigma^{2}$. Thus, the SINR at the receiver for $\mathrm{CU} i$ or D2D pair $j$ can be expressed as

$$
\begin{aligned}
& \gamma_{i}=\frac{p_{i} \cdot\left|g_{i, B}\right|^{2}}{\sigma^{2}+p_{j} \cdot\left|h_{j, B}\right|^{2}}, \\
& \gamma_{j}=\frac{p_{j} \cdot\left|g_{j}\right|^{2}}{\sigma^{2}+p_{i} \cdot\left|h_{i, j}\right|^{2}} .
\end{aligned}
$$

When the normalized bandwidth is employed, the achievable rate for $\mathrm{CU} i$ and $\mathrm{D} 2 \mathrm{D}$ pair $j$ can be written as

$$
\begin{aligned}
R_{i} & =\log _{2}\left(1+\gamma_{i}\right) \\
& =\log _{2}\left(1+\frac{p_{i} \cdot\left|g_{i, B}\right|^{2}}{\sigma^{2}+p_{j} \cdot\left|h_{j, B}\right|^{2}}\right), \\
R_{j} & =\log _{2}\left(1+\gamma_{j}\right) \\
& =\log _{2}\left(1+\frac{p_{j} \cdot\left|g_{j}\right|^{2}}{\sigma^{2}+p_{i} \cdot\left|h_{i, j}\right|^{2}}\right) .
\end{aligned}
$$

\section{Fair resource reuse for CU and D2D pair}

\subsection{Fairness index and optimization problem}

The system throughput is the primary performance index of the scheduling strategy for most wireless communication systems. And because of the radio spectrum rarity, some efforts need to be paid to achieve the potential throughput. The system fairness enables all the users to obtain indiscriminating services, which is also another important key index for wireless systems. The classical proportional fairness research is focused on the cellular network with one BS and multiple users, where there are $N$ active users and each user has the unlimited amount of data to transmit. Let $R_{i}(t)$ denote the achievable rate for $\mathrm{CU} i$ in slot $t$, and $T_{i}(t-1)$ indicate the scheduled average rate for $\mathrm{CU} i$ till slot $t-1$ as

$$
T_{i}(t-1)=\frac{\sum_{\tau=1}^{t-1} \hat{R}_{i}(\tau)}{(t-1)},
$$

where $R_{i}(t)=R_{i}(t)$ if CU $i$ is scheduled in slot $t$, else $R_{i}$ $(t)=0$. Let $R_{i}(t) / T_{i}(t-1)$ denote the relative achievable rate for $\mathrm{CU} i$ in slot $t$, and the $\mathrm{CU}$ with the maximum relative achievable rate, $\mathrm{CU} i$, is chosen for the transmission as $[19,20]$

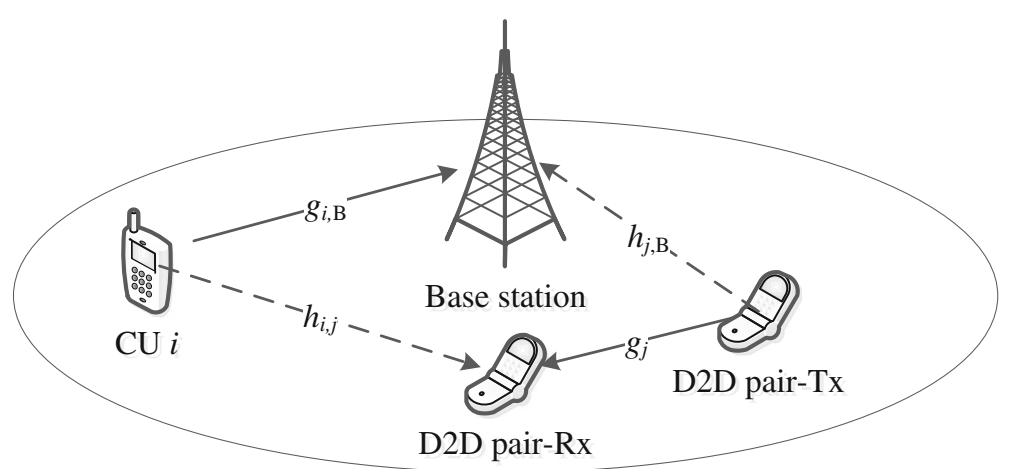

Fig. 1 System model of uplink resource reusing for some CU and D2D pair 


$$
i^{*}=\arg \max _{i} \frac{R_{i}(t)}{T_{i}(t-1)} .
$$

To avoid denominator zero, the initial value $T_{i}(0)$ is configured as the value close to zero, e.g., $10^{-8}$. In a similar way, the proportional fairness scheduling for the D2D pairs selects D2D pair $j "$ for the transmission as

$$
j^{*}=\arg \max _{j} \frac{R_{j}(t)}{T_{j}(t-1)},
$$

where $T_{j}(t-1)$ denotes the scheduled average rate for D2D pair $j$ till slot $t-1$ and $R_{j}(t) / T_{j}(t-1)$ denotes the relative achievable rate for D2D pair $j$ in slot $t$. Suppose the subchannel is reused by $\mathrm{CU} i$ and D2D pair $j$, let $\alpha_{i}(t)=1 / T_{i}(t-1)$ and $\beta_{j}(t)=1 / T_{j}(t-1)$, and for the expression to be convincing, we omit the parameter $t$, then the sum of relative achievable rate of both $\mathrm{CU} i$ and D2D pair $j$ can be expressed as

$$
\begin{aligned}
& R\left(p_{i}, p_{j}\right)=\alpha_{i} \cdot R_{i}+\beta_{j} \cdot R_{j} \\
& =\alpha_{i} \cdot \log _{2}\left(1+\gamma_{i}\right) \\
& \quad+\beta_{j} \cdot \log _{2}\left(1+\gamma_{j}\right) \\
& =\alpha_{i} \cdot \log _{2}\left(1+\frac{p_{i} \cdot\left|g_{i, B}\right|^{2}}{\sigma^{2}+p_{j} \cdot\left|h_{j, B}\right|^{2}}\right) \\
& \quad+\beta_{j} \cdot \log _{2}\left(1+\frac{p_{j} \cdot\left|g_{j}\right|^{2}}{\sigma^{2}+p_{i} \cdot\left|h_{i, j}\right|^{2}}\right) .
\end{aligned}
$$

Thus, the overall throughput optimization problem for one $\mathrm{CU}$ vs. one D2D pair can be formulated as follows:

$$
R\left(p_{i}^{*}, p_{j}^{*}\right)=\max R\left(p_{i}, p_{j}\right)
$$

subject to

$$
\begin{aligned}
& p_{i} \leq p_{i, \max }, C_{i} \in \mathbb{C}, \\
& p_{j} \leq p_{j, \max }, D_{j} \in \mathbb{D}, \\
& \gamma_{i} \geq \gamma_{i, \min }, C_{i} \in \mathbb{C}, \\
& \gamma_{j} \geq \gamma_{j, \min }, D_{j} \in \mathbb{D} .
\end{aligned}
$$

Constraints (6a) and (6b) guarantee that the transmit powers of $\mathrm{CU} i$ and D2D pair $j$ are within the maximum limit, denoted by $p_{i, \max }$ and $p_{j, \max }$, respectively. Constraints (6c) and (6d) represent the QoS requirement, i.e., guarantee the minimum data rate corresponding to the given service and the signal needs to satisfy with the minimum SINR value for $\mathrm{CU} i$ and $\mathrm{D} 2 \mathrm{D}$ pair $j$, expressed by $\gamma_{i, \min }$ and $\gamma_{j, \min }$, respectively. When reusing the resource by $\mathrm{CU} i$ and $\mathrm{D} 2 \mathrm{D}$ pair $j$, the range of the transmit power $p_{i}$ and $p_{j}$ based on constraints (6a)-(6d) forms the admissible area. The feasible domain for this optimization problem is further discussed in the next subsection. Therefore, the optimization problem (6) is to seek the optimal transmit power $p_{i}^{*}$ and $p_{j}^{*}$. The low complexity PFS algorithm is proposed to solve the problem, which is split into several phases.

(1) According to constraints (6a)-(6d), compute the admissible area for $\mathrm{CU} i$ and D2D pair $j$ when resource reusing occurs. If there exists such $p_{i}$ and $p_{j}$ to satisfy the aforementioned constraints, step into (2); else $\mathrm{CU} i$ and D2D pair $j$ cannot be reused.

(2) Seek the optimal power combination $\left(p_{i}^{*}, p_{j}^{*}\right)$ to make $R\left(p_{i}^{*}, p_{j}^{*}\right)=\max R\left(p_{i}, p_{j}\right)$.

\subsection{Admission control for CU vs. D2D pair}

Now, we analyze the feasible domain of the optimization problem in terms of constraints (6a) and (6d). Firstly, we assume no interference between CU $i$ and D2D pair $j$ because they employ orthogonal resources. According to (1a) and (1b), the minimum transmit powers for $\mathrm{CU} i$ and D2D pair $j$ can be written as

$$
\begin{aligned}
& p_{i, \min }=\frac{\gamma_{i, \min } \cdot \sigma^{2}}{\left|g_{i, B}\right|^{2}}, \\
& p_{j, \text { min }}=\frac{\gamma_{j, \min } \cdot \sigma^{2}}{\left|g_{j}\right|^{2}} .
\end{aligned}
$$

Substituting (1a) and (1b) into (6c) and (6d), and we achieve

$$
\begin{aligned}
\gamma_{i}= & \frac{p_{i} \cdot\left|g_{i, B}\right|^{2}}{\sigma^{2}+p_{j} \cdot\left|h_{j, B}\right|^{2}} \geq \gamma_{i, \min } \Rightarrow p_{i} \geq \frac{\gamma_{i, \min } \cdot \sigma^{2}}{\left|g_{i, B}\right|^{2}}+p_{j} \cdot \frac{\gamma_{i, \min } \cdot\left|h_{j, B}\right|^{2}}{\left|g_{i, B}\right|^{2}}, \\
\gamma_{j}= & \frac{p_{j} \cdot\left|g_{j}\right|^{2}}{\sigma^{2}+p_{i} \cdot\left|h_{i, j}\right|^{2}} \geq \gamma_{j, \min } \Rightarrow p_{i} \leq-\frac{\gamma_{j, \min } \cdot \sigma^{2}}{\gamma_{j, \min } \cdot\left|h_{i, j}\right|^{2}} \\
& +p_{j} \cdot \frac{\left|g_{j}\right|^{2}}{\gamma_{j, \min } \cdot\left|h_{i, j}\right|^{2}} .
\end{aligned}
$$

And we take (7a) and (7b) into (8a) and (8b), we have

$$
p_{i} \geq p_{i, \min }+p_{j} \cdot \frac{\gamma_{i, \min } \cdot\left|h_{j, B}\right|^{2}}{\left|g_{i, B}\right|^{2}}
$$




$$
p_{i} \leq-\frac{\left|g_{j}\right|^{2}}{\gamma_{j, \min } \cdot\left|h_{i, j}\right|^{2}} \cdot p_{j, \min }+p_{j} \cdot \frac{\left|g_{j}\right|^{2}}{\gamma_{j, \min } \cdot\left|h_{i, j}\right|^{2}} .
$$

As illustrated in Fig. 2a-d, the two lines, denoted by $\mathrm{I}_{1}$ and $I_{2}$, correspond to (9a) and (9b) with equality, respectively. The area above line $\mathrm{I}_{1}$ is where the minimum SINR for CU $i$ is satisfied. The area on the right of line $\mathrm{I}_{2}$ is where the minimum SINR for D2D pair $j$ is satisfied.

If the lines $I_{1}$ and $I_{2}$ have an intersection in the first quarter, denoted by point $\mathrm{A}$, the slope of $\mathrm{I}_{2}$ must be larger than that of $I_{1}$, that is

$$
\frac{\gamma_{i, \min } \cdot\left|h_{j, B}\right|^{2}}{\left|g_{i, B}\right|^{2}}<\frac{\left|g_{j}\right|^{2}}{\gamma_{j, \min } \cdot\left|h_{i, j}\right|^{2}} .
$$

According to (9a), (9b), and (10), we can get the transmit power at point $\mathrm{A}$ as

$$
p_{i}=\frac{\left(\left|g_{j}\right|^{2} \cdot \gamma_{i, \min }+\left|h_{j, B}\right|^{2} \cdot \gamma_{i, \min } \cdot \gamma_{j, \min }\right) \cdot \sigma^{2}}{\left|g_{i, B}\right|^{2} \cdot\left|g_{j}\right|^{2}-\left|h_{j, B}\right|^{2} \cdot\left|h_{i, j}\right|^{2} \cdot \gamma_{i, \min } \cdot \gamma_{j, \min }}
$$

$$
p_{j}=\frac{\left(\left|g_{i, B}\right|^{2} \cdot \gamma_{j, \min }+\left|h_{i, j}\right|^{2} \cdot \gamma_{i, \min } \cdot \gamma_{j, \min }\right) \cdot \sigma^{2}}{\left|g_{i, B}\right|^{2} \cdot\left|g_{j}\right|^{2}-\left|h_{j, B}\right|^{2} \cdot\left|h_{i, j}\right|^{2} \cdot \gamma_{i, \min } \cdot \gamma_{j, \min }}
$$

Together with the maximum transmit power limitation in (6a) and (6b), point A should fall in the square area, which denotes the maximum transmit power constraints in (6a) for CU $i$ and in (6b) for D2D pair $j$. And thus the admissible conditions will be

$$
\begin{aligned}
0 & <\frac{\left(\left|g_{j}\right|^{2} \cdot \gamma_{i, \min }+\left|h_{j, B}\right|^{2} \cdot \gamma_{i, \min } \cdot \gamma_{j, \min }\right) \cdot \sigma^{2}}{\left|g_{i, B}\right|^{2} \cdot\left|g_{j}\right|^{2}-\left|h_{j, B}\right|^{2} \cdot\left|h_{i, j}\right|^{2} \cdot \gamma_{i, \min } \cdot \gamma_{j, \min }} \\
& <p_{i, \max }, \\
0 & <\frac{\left(\left|g_{i, B}\right|^{2} \cdot \gamma_{j, \min }+\left|h_{i, j}\right|^{2} \cdot \gamma_{i, \min } \cdot \gamma_{j, \min }\right) \cdot \sigma^{2}}{\left|g_{i, B}\right|^{2} \cdot\left|g_{j}\right|^{2}-\left|h_{j, B}\right|^{2} \cdot\left|h_{i, j}\right|^{2} \cdot \gamma_{i, \min } \cdot \gamma_{j, \min }} \\
& <p_{j, \max } \cdot
\end{aligned}
$$

Observing Fig. 2a, point $\mathrm{A}$ is outside of the square area, which represents $\mathrm{CU} i$ and D2D pair $j$ cannot share the same resource based on constraints $(6 a)-(6 d)$. In Fig. $2 b-d$, point $A$ is within the square area and, any point in the shaded area, denoted by the transmit
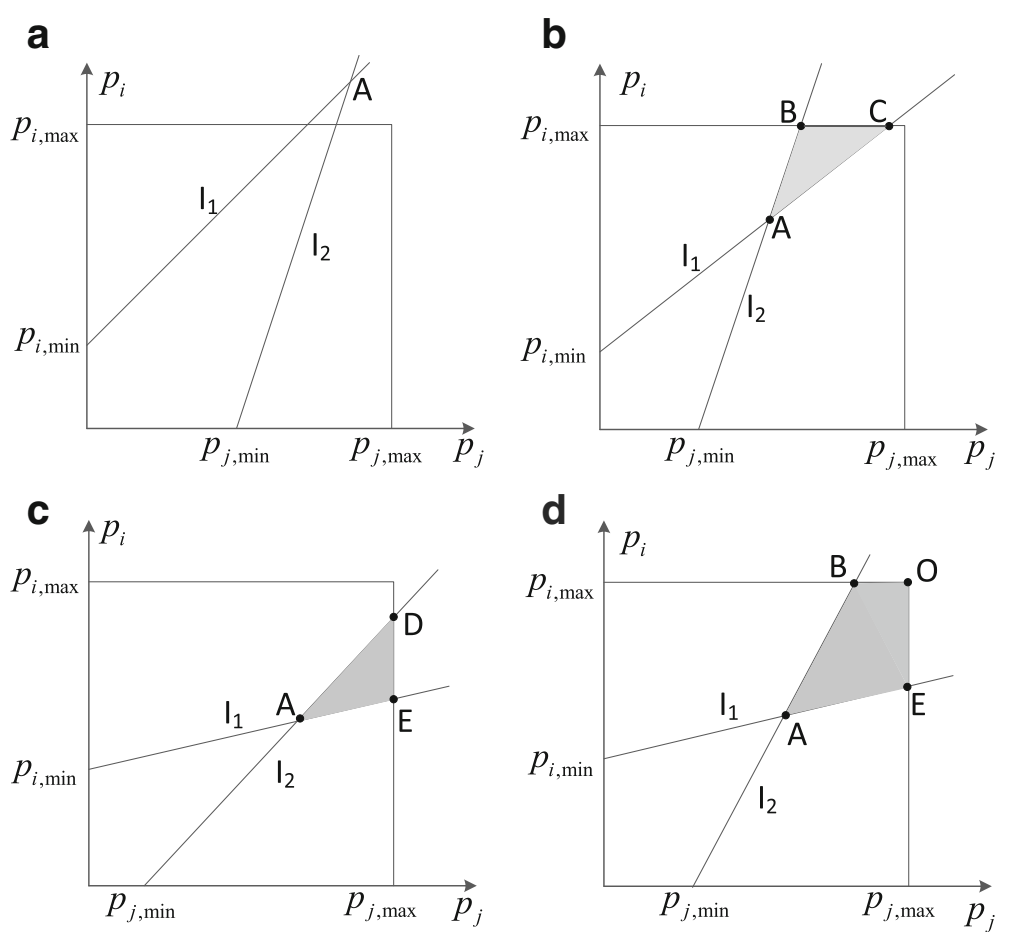

Fig. 2 Admissible area for the transmit power of CU $i$ and D2D pair j. a Without admissible area. b With admissible area (scenario 1). c With admissible area (scenario 2). $\mathbf{d}$ With admissible area (scenario 3) 
power for $\mathrm{CU} i$ and D2D pair $j$, satisfies constraints (6a)-(6d). Therefore, the shaded area is called the admissible area.

\subsection{Optimal transmit power control for CU vs. D2D pair}

The admissible area is denoted by $\Omega$, corresponding to the shadow region of the three possible scenarios represented in Fig. $2 \mathrm{~b}-\mathrm{d}$. Here, we investigate how to allocate power for the $\mathrm{CU}$ and for its reuse partner, D2D transmitter, to maximize the relative achievable rate, i.e., seek the optimal power combination $\left(p_{i}^{*}, p_{j}^{*}\right)$ within $\Omega$ to make $R\left(p_{i}^{*}, p_{j}^{*}\right)=\max R\left(p_{i}, p_{j}\right)$. It is demonstrated in this paper that only several power combinations need to be computed and compared, and thus the optimal solution can be achieved, which avoids the complex optimization algorithms.

Proposition 1. When $\mathrm{CU} i$ and D2D pair $j$ reuse the resource, the optimal transmit power satisfies $p_{i}^{*}$ $=p_{i, \max }$ or $p_{j}^{*}=p_{j, \max }[21]$.

Proof: Let $\mu>1$ and according to (5), we have

$$
\begin{aligned}
R\left(\mu p_{i}, \mu p_{j}\right)= & \alpha_{i} \cdot \log _{2}\left(1+\frac{\mu \cdot p_{i} \cdot\left|g_{i, B}\right|^{2}}{\sigma^{2}+\mu \cdot p_{j} \cdot\left|h_{j, B}\right|^{2}}\right) \\
& +\beta_{j} \cdot \log _{2}\left(1+\frac{\mu \cdot p_{j} \cdot\left|g_{j}\right|^{2}}{\sigma^{2}+\mu \cdot p_{i} \cdot\left|h_{i, j}\right|^{2}}\right) \\
= & \alpha_{i} \cdot \log _{2}\left(1+\frac{p_{i} \cdot\left|g_{i, B}\right|^{2}}{\frac{\sigma^{2}}{\mu}+p_{j} \cdot\left|h_{j, B}\right|^{2}}\right) \\
& +\beta_{j} \cdot \log _{2}\left(1+\frac{p_{j} \cdot\left|g_{j}\right|^{2}}{\frac{\sigma^{2}}{\mu}+p_{i} \cdot\left|h_{i, j}\right|^{2}}\right) \\
> & R\left(p_{i}, p_{j}\right) .
\end{aligned}
$$

Hence, the solution of (6) will have $p_{i}^{*}=p_{i, \max }$ or $p_{j}^{*}$ $=p_{j, \max }$, i.e., the optimal power combination occurs in the border of $\Omega$.

Proposition 2. Under the premise of $p_{j}=p_{j, \max }$ or $p_{i}=$ $p_{i \text {, max }}$, i.e., one variable is constant, the function $R\left(p_{i}, p_{j}\right)$ is convex.

Proof: According to (5), and we have

$$
\begin{aligned}
& R\left(p_{i}, p_{j}\right)= \alpha_{i} \cdot \log _{2}\left(1+\frac{p_{i} \cdot\left|g_{i, B}\right|^{2}}{\sigma^{2}+p_{j} \cdot\left|h_{j, B}\right|^{2}}\right) \\
&+\beta_{j} \cdot \log _{2}\left(1+\frac{p_{j} \cdot\left|g_{j}\right|^{2}}{\sigma^{2}+p_{i} \cdot\left|h_{i, j}\right|^{2}}\right) \\
&=\beta_{j} \cdot\left[\frac{\alpha_{i}}{\beta_{j}} \cdot \log _{2}\left(1+\frac{p_{i} \cdot\left|g_{i, B}\right|^{2}}{\sigma^{2}+p_{j} \cdot\left|h_{j, B}\right|^{2}}\right)+\log _{2}\left(1+\frac{p_{j} \cdot\left|g_{j}\right|^{2}}{\sigma^{2}+p_{i} \cdot\left|h_{i, j}\right|^{2}}\right)\right] \\
&=\beta_{j} \cdot \log _{2}\left[\left(1+\frac{p_{i} \cdot\left|g_{i, B}\right|^{2}}{\sigma^{2}+p_{j} \cdot\left|h_{j, B}\right|^{2}}\right)^{\frac{\alpha_{i}}{\beta_{j}}} \cdot\left(1+\frac{p_{j} \cdot\left|g_{j}\right|^{2}}{\sigma^{2}+p_{i} \cdot\left|h_{i, j}\right|^{2}}\right)\right] .
\end{aligned}
$$

Without losing the generality, if $\alpha_{i} \geq \beta_{j}$, let $v=\alpha_{i} / \beta_{j}$, and $v \geq 1$ can be achieved; else if $\alpha_{i}<\beta_{j}$, let $v=\beta_{j} / \alpha_{i}$. The twobased logarithm is monotonically increasing function, let

$$
Q\left(p_{i}, p_{j, \max }\right)=\left(1+\frac{p_{i} \cdot\left|g_{i, B}\right|^{2}}{\sigma^{2}+p_{j, \max } \cdot\left|h_{j, B}\right|^{2}}\right)^{v} \cdot\left(\frac{1+p_{j, \max } \cdot\left|g_{j}\right|^{2}}{\sigma^{2}+p_{i} \cdot\left|h_{i, j}\right|^{2}}\right),
$$

By differentiating $Q\left(p_{i}, p_{j, \max }\right)$ with respect to $p_{i}$ we can get

$$
\frac{d Q}{d p_{i}}=\frac{C \cdot p_{i}^{2}+D \cdot p_{i}+E}{\left(\sigma^{2}+p_{j, \max } \cdot\left|h_{j, B}\right|^{2}\right) \cdot\left(\sigma^{2}+p_{i} \cdot\left|h_{i, j}\right|^{2}\right)^{2}},
$$

where

$$
\begin{aligned}
C= & v \cdot\left|g_{i, B}\right|^{2} \cdot\left(\left|h_{i, j}\right|^{2}\right)^{2}, \\
D= & 2 v \cdot\left|g_{i, B}\right|^{2} \cdot\left|h_{i, j}\right|^{2} \cdot \sigma^{2}+(v-1) \cdot p_{j, \max } \cdot\left|g_{i, B}\right|^{2} \cdot\left|g_{j}\right|^{2} \cdot\left|h_{i, j}\right|^{2}, \\
E= & -p_{j, \max } \cdot\left|g_{j}\right|^{2} \cdot\left|h_{i, j}\right|^{2} \cdot\left(\cdot \sigma^{2}+p_{j, \max } \cdot\left|h_{j, B}\right|^{2}\right)+v \cdot\left|g_{i, B}\right|^{2} \cdot \sigma^{2} . \\
& \left(\sigma^{2}+p_{j, \max } \cdot\left|g_{j}\right|^{2}\right)
\end{aligned}
$$

Since denominator of (14) is always positive, if $\frac{d Q}{d p_{i}}=0$ , we can achieve $C \cdot p_{i}^{2}+D \cdot p_{i}+E=0$. To solve such the quadratic equation with one unknown, $p_{i}=\frac{1}{2 C}$ $\left(-D \pm \sqrt{D^{2}-4 C E}\right)$ can be obtained. The range of the parameter $p_{i} \in\left[0, p_{i, \max }\right]$, i.e., a real and non-negative $p_{i}$ can only occur for $E \leq 0$, since $C>0$ and $D>0$. Denote such a $p_{i}$ by $p_{i}^{\text {rp }}$. To decide $p_{i}^{\text {rp }}$ corresponds to a maximum or minimum for $Q\left(p_{i}, p_{j, \max }\right)$, we calculate the second derivative as follows: 
Zheng et al. EURASIP Journal on Wireless Communications and Networking (2017) 2017:170

Page 7 of 12

$\frac{d^{2} Q}{d p_{i}{ }^{2}}=\frac{p_{j, \max } \cdot\left|g_{j}\right|^{2} \cdot\left|h_{i, j}\right|^{2} \cdot\left[2\left|h_{i, j}\right|^{2} \cdot\left(\sigma^{2}+p_{j, \max } \cdot\left|h_{j, B}\right|^{2}\right)-(1+v) \cdot\left|g_{i, B}\right|^{2} \cdot \sigma^{2}\right]}{\left(\sigma^{2}+p_{j, \max } \cdot\left|h_{j, B}\right|^{2}\right) \cdot\left(\sigma^{2}+p_{i} \cdot\left|h_{i, j}\right|^{2}\right)^{3}}$.

Since $E \leq 0$, we have

$p_{j, \max } \cdot\left|g_{j}\right|^{2} \cdot\left|h_{i, j}\right|^{2} \cdot\left(\sigma^{2}+p_{j, \max } \cdot\left|h_{j, B}\right|^{2}\right) \geq v \cdot\left|g_{i, B}\right|^{2} \cdot \sigma^{2}$ $\cdot\left(\sigma^{2}+p_{j, \max } \cdot\left|g_{j}\right|^{2}\right)$. Together with the aforementioned assumption of $v \geq 1$, we have

$$
\begin{gathered}
2 p_{j, \max } \cdot\left|g_{j}\right|^{2} \cdot\left|h_{i, j}\right|^{2} \cdot\left(\sigma^{2}+p_{j, \max } \cdot\left|h_{j, B}\right|^{2}\right) \geq(1+v) \cdot\left|g_{i, B}\right|^{2} \\
\cdot \sigma^{2} \cdot\left(\sigma^{2}+p_{j, \max } \cdot\left|g_{j}\right|^{2}\right)
\end{gathered}
$$

Dividing by $p_{j, \max } \cdot\left|g_{j}\right|^{2}$ on both sides of (16), we obtain

$$
\begin{aligned}
2\left|h_{i, j}\right|^{2} & \left(\sigma^{2}+p_{j, \max } \cdot\left|h_{j, B}\right|^{2}\right) \geq(1+v) \cdot\left|g_{i, B}\right|^{2} \cdot \sigma^{2} \\
& \left(1+\frac{\sigma^{2}}{p_{j, \max } \cdot\left|g_{j}\right|^{2}}\right) \geq(1+v) \cdot\left|g_{i, B}\right|^{2} \cdot \sigma^{2} .
\end{aligned}
$$

Comparing (17) with (15), we have $\frac{d^{2} Q}{d p_{i}{ }^{2}} \geq 0 . Q\left(p_{i}, p_{j, \max }\right)$ is then convex with respect to $0 \leq p_{i} \leq p_{i, \max }$ and $p_{i}^{\mathrm{rP}}$ is a minimum point for $Q\left(p_{i}, p_{j, \max }\right)$. Due to symmetry, the aforementioned result also applies for $p_{j}$. According to the three scenarios illustrated in Fig. $2 b-d$, the optimal power combination $\left(p_{i}^{*}, p_{j}^{*}\right)$ belongs to the predefined two or three points in the admissible area $\Omega$, compute the value according to (6), compare the two or three results and choose optimal power combination with the largest result as follows:

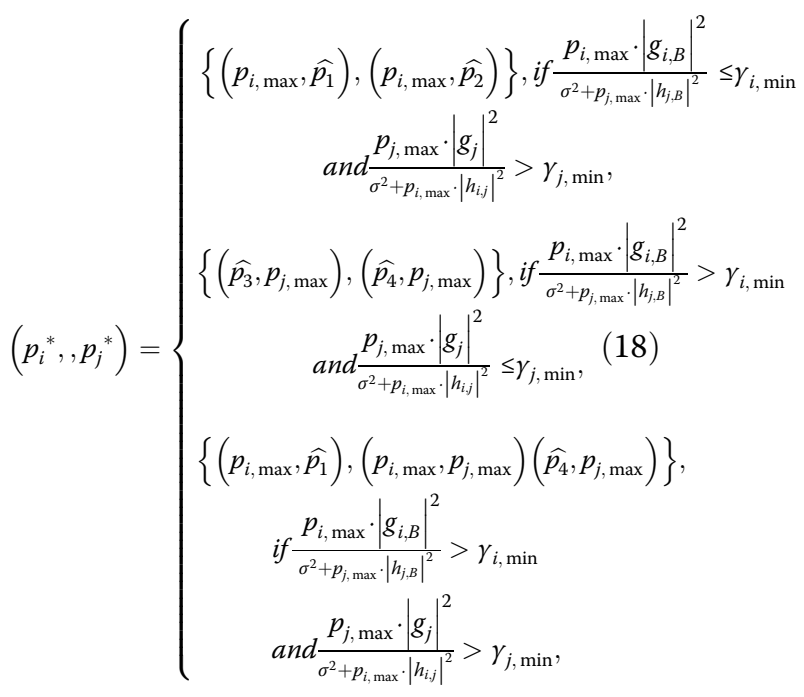

where

$$
\begin{aligned}
& \widehat{p_{1}}=\frac{\gamma_{j, \min } \cdot\left(\sigma^{2}+p_{i, \max } \cdot\left|h_{i, j}\right|^{2}\right)}{\left|g_{j}\right|^{2}}, \\
& \widehat{p_{2}}=\frac{p_{i, \max } \cdot\left|g_{i, B}\right|^{2}-\gamma_{i, \min } \cdot \sigma^{2}}{\gamma_{i, \min } \cdot\left|h_{j, B}\right|^{2}}, \\
& \widehat{p_{3}}=\frac{p_{j, \max } \cdot\left|g_{j}\right|^{2}-\gamma_{j, \min } \cdot \sigma^{2}}{\gamma_{j, \min } \cdot\left|h_{i, j}\right|^{2}},
\end{aligned}
$$

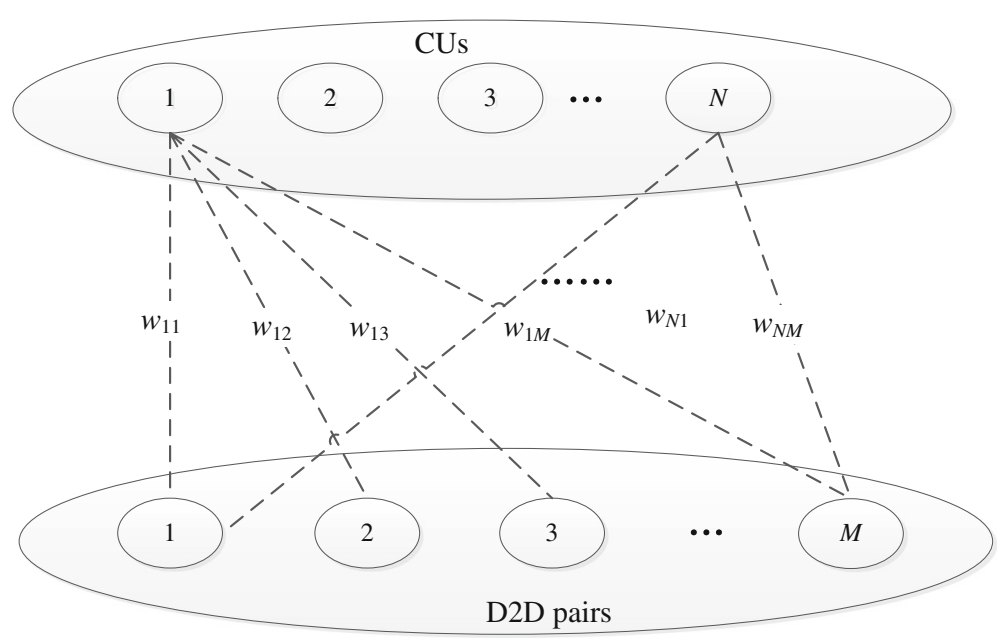

Fig. 3 Bipartite graph for CU and D2D pair matching 


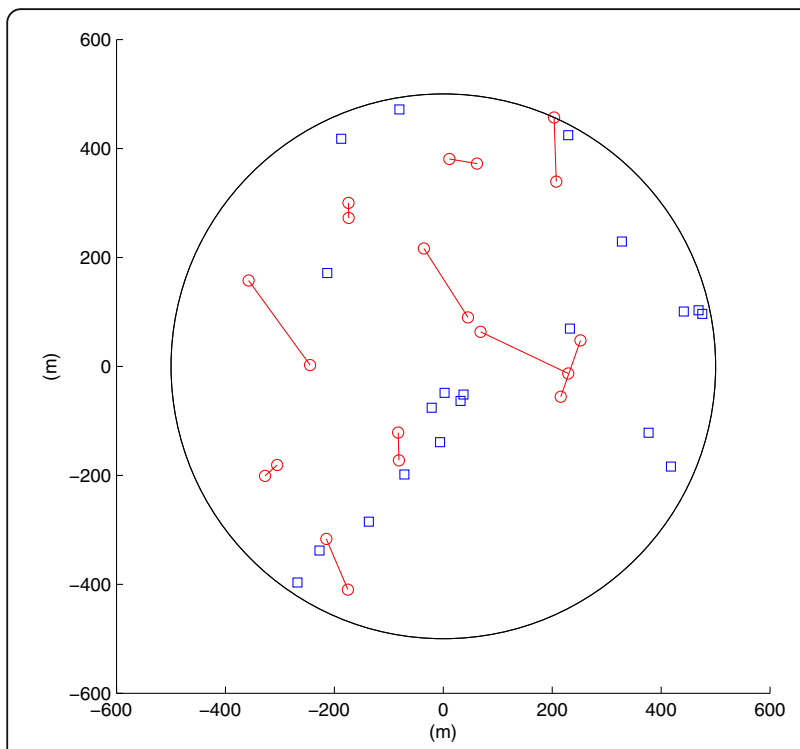

Fig. 4 An example of CU and D2D pair distribution in the cell

$$
\widehat{p_{4}}=\frac{\gamma_{i, \min } \cdot\left(p_{j, \max } \cdot\left|h_{j, B}\right|^{2}+\sigma^{2}\right)}{\left|g_{i, B}\right|^{2}} .
$$

Now, when $\mathrm{CU}$ reuses with $\mathrm{D} 2 \mathrm{D}$ pair, the optimal power combination $\left(p_{i}^{*}, p_{j}^{*}\right)$ satisfied with constraints (5a)-(5d) can be achieved.

\section{Resource matching for multiple CUs and D2D pairs}

In the mathematical field of graph theory, a bipartite graph is a graph whose vertices can be divided into two disjoint sets $\mathbb{U}$ and $\mathbb{V}$ (that is, $\mathbb{U}$ and $\mathbb{V}$ are each independent sets) such that every edge connects a vertex in $\mathbb{U}$ to one in $\mathbb{V}$. A bipartite graph is often denoted by $\mathbb{G}=(\mathbb{U}, \mathbb{V}, \mathbb{E})$, whose partition has the parts $\mathbb{U}$ and $\mathbb{V}$, with $\mathbb{E}$ denoting the edges of the graph. Compared with our proposed problem of reusing resources between $\mathrm{CU}$ and D2D pair, the set of active CUs, denoted by $\mathbb{C}$, and the set of D2D pairs, denoted by $\mathbb{D}$, are disjoint sets, we have $C \cap \mathbb{D}==\varnothing$. If $\mathrm{CU} i$ and D2D pair $j$ reuse the resource, there exists an edge to connect the vertex $C_{i}$ and $D_{j}$, which belong to $\mathbb{C}$ and $\mathbb{D}$, respectively; else there is no edge between $C_{i}$ and $D_{j}$. Therefore, the problem can be expressed by a bipartite graph.

The optimization objective described in (6) realizes the matching for one $\mathrm{CU}$ and one D2D pair. When $K$ subchannels are employed, the resource reuse for $N$ active CUs and $M$ pairs of D2D users is to solve the following optimization problem:

$$
\max \sum \rho_{i, j} \cdot w_{i, j}=\max \sum \rho_{i, j} \cdot R\left(p_{i}^{*}, p_{j}^{*}\right) .
$$

Subject to

$$
\sum_{j} \rho_{i, j} \leq 1, \rho_{i, j} \in\{0,1\}, C_{i} \in \mathbb{C}
$$

\begin{tabular}{|c|c|}
\hline Parameter & Value \\
\hline Cell radius & $500 \mathrm{~m}$ \\
\hline Maximum distance for the D2D pair & $200 \mathrm{~m}$ \\
\hline Carrier frequency $\left(f_{c}\right)$ & $2 \mathrm{GHz}$ \\
\hline Uplink bandwidth & $10 \mathrm{MHz}$ \\
\hline Number of sub-channels & 10 \\
\hline Maximum transmit (Tx) power & $\begin{array}{l}23 \mathrm{dBm} \\
P L_{\text {LOS }}=40 \log _{10}(d)+7.56-17.3 \log _{10}\left(h_{1}^{\prime}\right)-17.3 \log _{10}\left(h_{2}^{\prime}\right)+2.7 \log _{10}\left(f_{c}\right)\end{array}$ \\
\hline \multirow{2}{*}{ Path loss model } & $\begin{array}{l}P L_{\text {NLOS }}=\left(44.9-6.55 \log _{10}\left(h_{1}\right)\right) \cdot \log _{10}(d)+5.83 \log _{10}\left(h_{1}\right)+9.78+34.97 \log _{10}\left(f_{c}\right) \\
\text { where the probability of LOS can be expressed by }\end{array}$ \\
\hline & $\begin{array}{l}P_{\text {LOS }}=\min \left(\frac{18}{d}, 1\right) \cdot\left(1-e^{-\frac{d}{36}}\right)+e^{-\frac{d}{36}} \\
\text { (1) Cellular communication: } \\
\text { The distance between CU and BS is denoted by } d_{;} h_{1}=10 \mathrm{~m}, h_{1}^{\prime}=9 \mathrm{~m} \text {, and } h_{2}^{\prime}=0.5 \mathrm{~m} \\
\text { denote the antenna height of BS, the effective height of } \mathrm{BS} \text {, } \\
\text { and the effective height of CU, respectively; } \\
\text { (2) D2D communication: } \\
\text { The distance between D2D pair is denoted by } d_{;} h_{1}=1.5 \mathrm{~m}, h_{1}^{\prime}=0.5 \mathrm{~m} \text { and } h_{2}^{\prime}=0.5 \mathrm{~m} \\
\text { denote the antenna height of D2D receiver, the effective height of D2D receiver, } \\
\text { and the effective height of D2D transmitter, respectively. }\end{array}$ \\
\hline Shadowing & Log-normal distribution with standardization deviation $7 \mathrm{~dB}$ \\
\hline Multiple-path fading & Rayleigh distribution \\
\hline
\end{tabular}

Table 1 Simulation parameters 

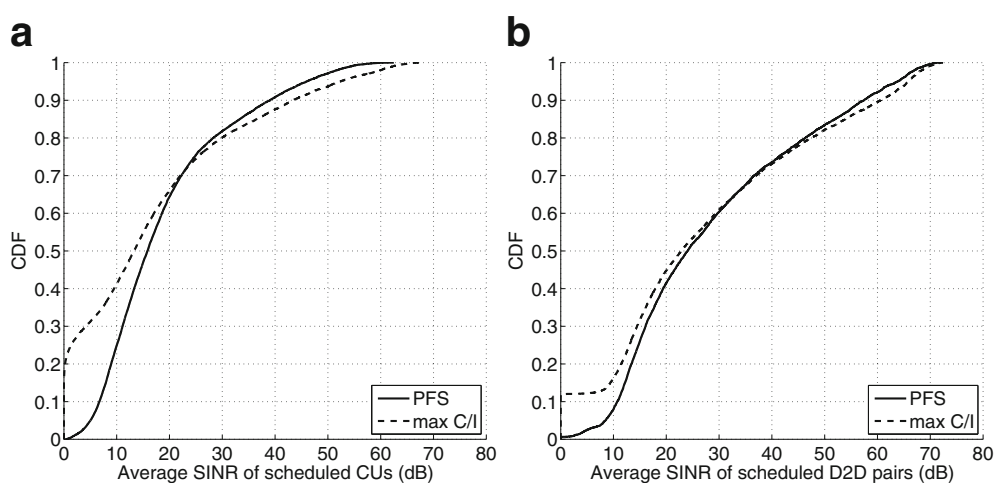

Fig. 5 CDF of average SINR for PFS and max C/I. a CUs. b D2D pairs

$$
\begin{aligned}
& \sum_{i} \rho_{i, j} \leq 1, \rho_{i, j} \in\{0,1\}, D_{j} \in \mathbb{D} . \\
& \sum \rho_{i, j} \leq K, \rho_{i, j} \in\{0,1\}, C_{i} \in \mathbb{C}, D_{j} \in \mathbb{D}
\end{aligned}
$$

Constraints (19a) and (19b) mean one CU only reuses the resource with one D2D pair and vice versa; $\rho_{i, j}$ indicates whether $\mathrm{CU} i$ and D2D pair $j$ reuse the resource or not; if reuse, $\rho_{i, j}=1$; else, $\rho_{i, j}=0$. Constraint (19c) indicates that most $K$ subchannels can be shared by the CUs and D2D pairs. Therefore, the optimization problem described in (19) can be modeled as the matching problem of weighted bipartite graph, as shown in Fig. 3. CUs and D2D pairs belong to two disjoint sets, denoted by $(\mathbb{C}, \mathbb{D})$. In the cell, the number of D2D pairs is usually smaller than that of CUs, i.e., $M<N$. When $\mathrm{CU} i$ and D2D pair $j$ reuse the resource, the edge connecting them can be expressed as $\left\langle C_{i}, D_{j}\right\rangle$ with weight $w_{i, j}$, which is the maximum relative achievable rate corresponding to the optimal power combination $\left(p_{i}^{*}, p_{j}^{*}\right)$ computed by (5).
The classic Kuhn-Munkres (KM) algorithm [22] can be employed to solve the aforementioned bipartite graph maximum weight matching.

\section{Numerical results}

We consider a single cell network, where 20 conventional CUs and 10 pairs of D2D users are uniformly distributed in the cell and an example is illustrated in Fig. 4, where the large circle denoted the cell with the radius $500 \mathrm{~m}$ and the square point and the circle point indicate $\mathrm{CU}$ and $\mathrm{D} 2 \mathrm{D}$ pair, respectively.

We assume the total bandwidth is $10 \mathrm{MHz}$, which is divided equally by 10 subchannels and each one is $1 \mathrm{MHz}$. The subchannel is occupied by reusing CUD2D pair or just CU alone, 2000 scheduling slots are employed and the 3GPP channel model is adopted [23]. Our simulation parameters are summarized in Table 1.

The same user distribution of CUs and D2D pairs is employed to keep performance comparable among different algorithms. Max C/I resource reusing algorithm for the CUs and D2D pairs aims to just improve the throughput [5]. The PFS algorithm proposed in this paper devotes to optimize both throughput and fairness
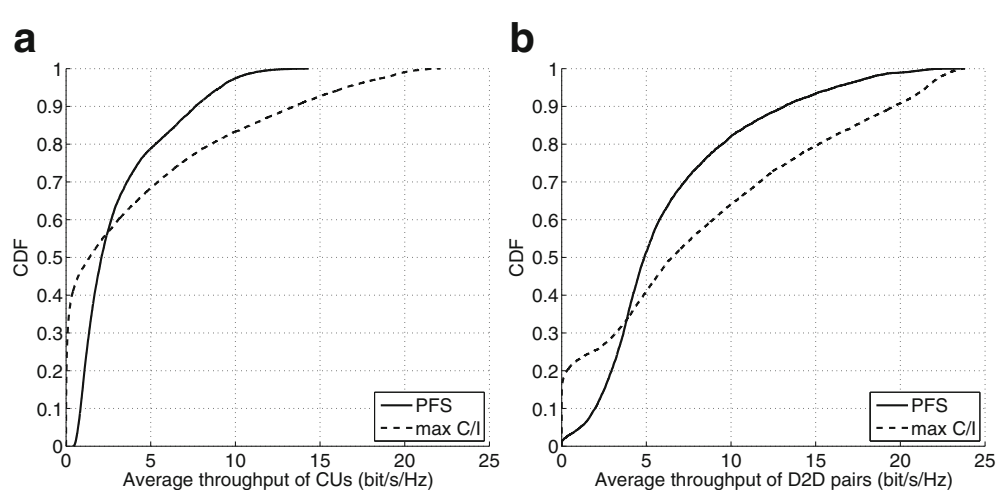

Fig. 6 CDF of average throughput for PFS and max C/I. a CUs. b D2D pairs 
Table 2 Numerical analysis for PFS and max C/I

\begin{tabular}{|c|c|c|c|c|c|c|}
\hline & \multicolumn{3}{|l|}{ PFS algorithm } & \multicolumn{3}{|l|}{ Max C/I algorithm } \\
\hline & $\begin{array}{l}\text { Average throughput } \\
\text { mean }\end{array}$ & $\begin{array}{l}\text { Average throughput } \\
\text { variance }\end{array}$ & $\begin{array}{l}\text { Average Tx power } \\
(\mathrm{dBm})\end{array}$ & $\begin{array}{l}\text { Average throughput } \\
\text { mean }\end{array}$ & $\begin{array}{l}\text { Average throughput } \\
\text { variance }\end{array}$ & $\begin{array}{l}\text { Average Tx power } \\
(\mathrm{dBm})\end{array}$ \\
\hline CUs & 3.18 & 2.69 & 22.78 & 4.20 & 5.51 & 22.98 \\
\hline $\begin{array}{l}\text { D2D } \\
\text { pairs }\end{array}$ & 6.29 & 4.47 & 21.43 & 8.17 & 7.05 & 22.41 \\
\hline
\end{tabular}

to make the users with bad channel conditions can also obtain the served opportunities, and thus improve the QoS of these users. As for the QoS requirement, the minimum data rate should be guaranteed, which corresponds to the minimum SINR threshold $0 \mathrm{~dB}$ in our simulation, the user has no transmit opportunity if its SINR is smaller than the threshold in a scheduling slot. To achieve the average SINR of a certain user, all the SINR values of the user are added up when it is scheduled and then divided by its number of scheduling counts. The cumulative distribution function (CDF) of the average SINR for the scheduled users is illustrated in Fig. 5.

In Fig. 5, it is indicated that more than 10\% CUs and D2D pairs are not served when max C/I algorithm is applied. As for PFS algorithm, the ratio is close to 0 for both CUs and D2D pairs. More users can get the serving opportunities, and thus, the fairness is improved.

By implementing 1000 times of user distribution and 2000 scheduling slots within each distribution, the CDF of the average throughput (bit/s/Hz) employed by PFS and max C/I algorithm for both CUs and D2D pairs are illustrated in Fig. 6, and the performance is summarized in Table 2.

The mean indicates the average throughput for all the CUs or D2D pairs within the 2000 scheduling slots for 1000 times of user distribution, and the variance denotes the deviation level from the mean value, where the smaller variance implies better fairness. PFS algorithm achieves the gain of fairness at the expenditure of some throughput. As for CUs, the improvement of fairness is about $51 \%$ while the loss of throughput is about 24\%; and for D2D pairs, 37 vs. $23 \%$. The average transmit power is also listed in Table 2, which indicates the similar power consumption for both algorithms.

To verify the performance gain of optimal transmit power control proposed in PFS algorithm, by implementing 1000 times of user distribution and 2000 scheduling slots within each distribution, the CDF of the average throughput employed by the optimal and maximum power for both CUs and D2D pairs are illustrated in Fig. 7, and the performance is summarized in Table 3.

Compared with the maximum power transmission, for CUs the PFS algorithm with the optimal power control can achieve 22\% fairness gain while yield 5.1\% throughput loss; For D2D pairs, it can achieve both $27 \%$ fairness gain and $17 \%$ throughput loss. It can be predicted that in the multi-cell environments the power control is much more important because of the extra interference from the adjacent cells.

There are 10 subchannels to schedule CUs and D2D pairs, when more users are distributed in the cell, the simulation results for $20 \mathrm{CUs}$ and $10 \mathrm{D} 2 \mathrm{D}$ pairs vs. 30 CUs and 15 D2D pairs are illustrated in Fig. 8. With the increasing number of users, the
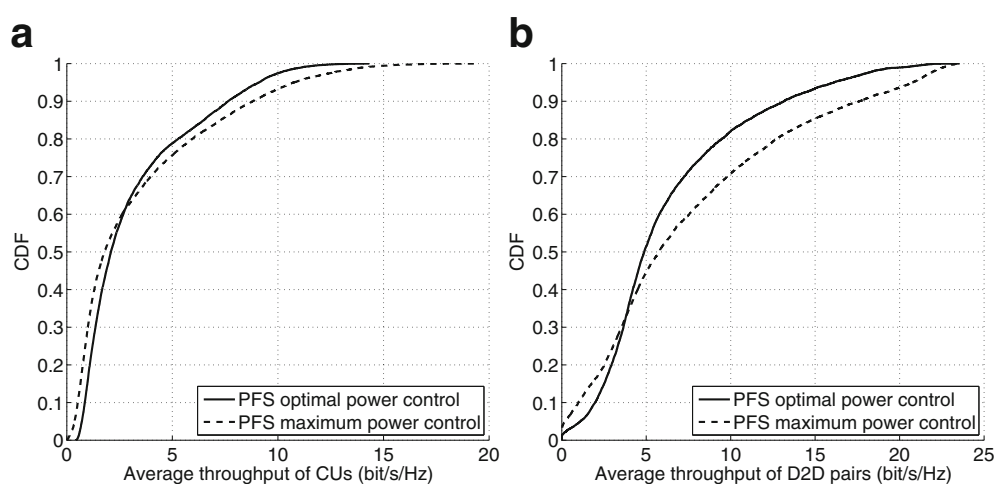

Fig. 7 The CDF of average throughput of PFS with different power control methods. a CUs. b D2D pairs 
Table 3 Numerical analysis for PFS with different power control methods

\begin{tabular}{llllllll}
\hline & \multicolumn{2}{l}{ PFS optimal power control } & & \multicolumn{3}{l}{ PFS maximum power control } \\
\cline { 2 - 3 } & $\begin{array}{l}\text { Average throughput } \\
\text { mean }\end{array}$ & $\begin{array}{l}\text { Average throughput } \\
\text { variance }\end{array}$ & $\begin{array}{l}\text { Average Tx power } \\
(\mathrm{dBm})\end{array}$ & $\begin{array}{l}\text { Average throughput } \\
\text { mean }\end{array}$ & $\begin{array}{l}\text { Average throughput } \\
\text { variance }\end{array}$ & $\begin{array}{l}\text { Average Tx power } \\
(\mathrm{dBm})\end{array}$ \\
\hline CUs & 3.18 & 2.69 & 22.78 & 3.35 & 3.44 & 23.00 \\
D2D & 6.29 & 4.47 & 21.43 & 7.61 & 6.09 & 23.00 \\
pairs & & & & &
\end{tabular}

performance of CUs is degraded greatly, more than $20 \%$ CUs lose the scheduling opportunity and nearly $60 \%$ CUs have a very low throughput; but for D2D pairs, the influence can be ignored.

The simulation results can be explained as follows. Because the distance between the D2D pair is shorter and LOS path existed with greater possibility, the channel gain between the D2D pair is usually better than that between the $\mathrm{CU}$ and the base station, and generally, the data rate of $\mathrm{D} 2 \mathrm{D}$ pair is larger than that of CU, which can be observed in Fig. 6. For PFS algorithm, the objective is to maximize the sum of relative achievable rate, denoted by $\max \left(\alpha_{i} R_{i}+\beta_{j} R_{j}\right)$, where the relative achievable rate of the D2D pair, $\beta_{j} R_{j}$, is the main contribution. Therefore, the transmit opportunities of CUs are blocked. In this sense, when more users, especially more D2D pairs, the proportional fairness becomes the proportional fairness dedicated for D2D pairs.

To avoid such a problem, in the practical communication systems, some measures may need to be adopted, including but not limited to

The D2D pairs can only be scheduled in certain subchannels, and some subchannels are dedicated for the scheduling of CUs;

- Limit the maximum transit power of D2D pairs, it may be realized by system broadcast information to indicate the D2D pairs in the cell. And the permitted transmit power of D2D pairs can be adjusted according to the traffic load in the cell.

As for the complexity of the algorithm, for the scenario of $N$ active CUs and $M$ pairs of D2D users, the complexity to compute the optimal power combination is $O(N \cdot M)$ and that of bipartite graph maximum weight matching employing $\mathrm{KM}$ algorithm is $O\left(N^{3}\right)$.

\section{Conclusions and discussions}

In this paper, we investigated on the scheduling for $\mathrm{CU}$ and D2D pair that share the uplink subchannel resource in the cellular network. In order to achieve the excellent tradeoff between throughput and fairness, the PFS algorithm is proposed based on the proportional fairness criteria, which divides into the admissible area decision, solving the optimal power combination, and utilizing bipartite graph for resource reuse in multiple subchannels. Several factors, e.g., sleeping feature and hybrid automatic repeat request process limitation, restrict the number of active users in a scheduling slot, and the complexity of PFS algorithm can be acceptable. The simulation results also verify that the PFS algorithm can guarantee the good throughput and fairness for both CUs and D2D pairs.

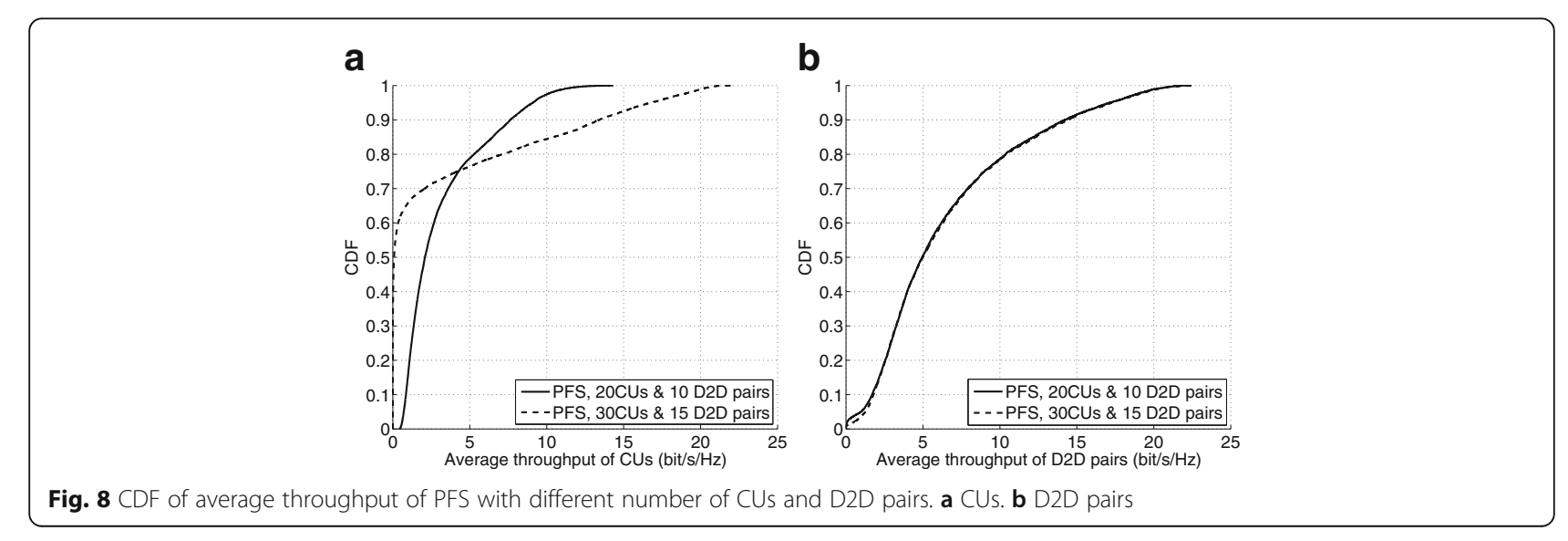




\section{Acknowledgements}

This work is supported by the Natural Science Foundation of Jiangsu Province of China under Grant BK21030874, the Natural Science Foundation of China under Grants 61372126 and 61302101, and Jinling Institute of Technology Project No. 2015-b-29.

\section{Authors' contributions}

WZ carried out the research on D2D communication, participated in the proposed algorithm design and the simulation, and drafted the manuscript. $\mathrm{JH}$ participated in the simulation. $\mathrm{CL}$ conceived of the study, participated in the algorithm design, and helped in drafting the manuscript. YF helped in drafting the manuscript. All authors read and approved the final manuscript.

\section{Competing interests}

The authors declare that they have no competing interests.

\section{Publisher's Note}

Springer Nature remains neutral with regard to jurisdictional claims in published maps and institutional affiliations.

\section{Author details}

${ }^{1}$ Jinling Institute of Technology, Nanjing 211169, China. ${ }^{2}$ Nanjing University of Posts and Telecommunications, Nanjing 210003, China.

Received: 10 April 2017 Accepted: 28 September 2017

Published online: 24 October 2017

\section{References}

1. P Schulz, M Matthe, H Klessig, et al., Latency critical loT applications in 5G: perspective on the design of radio interface and network architecture. IEEE Commun. Mag. 55(2), 70-78 (2017)

2. G Fodor, E Dahlman, G Mildh, et al., Design aspects of network assisted device-to-device communications. IEEE Commun. Mag. 50(3), 170-177 (2012)

3. $\mathrm{CH}$ Yu, O Tirkkonen, K Doppler, C Ribeiro, in Proc. IEEE Inter. Conf. Commun (ICC), Power Optimization of Device-to-Device Communication Underlaying Cellular Communication (IEEE, Dresden, 2009), pp. 1-5

4. C Xu, L Song, Z Han, Q Zhao, X Wang, X Cheng, B Jiao, Efficiency resource allocation for device-to-device underlay communication systems: a reverse iterative combinatorial auction based approach. IEEE J. Sel. Areas. Commun. 31(9), 348-358 (2013)

5. D Feng, L Lu, Y Wu, GY Li, G Feng, S Li, Device-to-device communications underlaying cellular networks. IEEE Trans. Commun. 61(8), 3541-3551 (2013)

6. CH Yu, K Doppler, CB Ribeiro, O Tirkkonen, Resource sharing optimization for device-to-device communication underlaying cellular networks. IEEE Trans. Wirel. Commun. 10(8), 2752-2763 (2011)

7. HJ Chou, RY Chang, Joint mode selection and interference management in device-to-device communications underlaid MIMO cellular networks. IEEE Trans. Wirel. Commun. 16(2), 1120-1134 (2017)

8. L Wang, H Tang, H Wu, GL Stuber, Resource allocation for D2D communications underlay in Rayleigh fading channels. IEEE Trans. Veh. Technol. 66(2), 1159-1170 (2017)

9. N Lee, X Lin, JG Andrews, RW Heath, Power control for D2D underlaid cellular networks: modeling, algorithms and analysis. IEEE J. Sel. Areas. Commun. 33(1), 1-13 (2015)

10. D Calabuig, JF Monserrat, N Cardona, Proportionally fair scheduler for heterogeneous wireless systems. Trans. Emerg. Tel. Technol. 23(1), 1-5 (2011)

11. M Ayhan, Y Zhao, HA Choi, in Proc. IEEE Global Commun. Conf. (GLOBECOM), Utilizing Geometric Mean in Proportional Fair Scheduling: Enhanced Throughput and Fairness in LTE DL (IEEE, Washington, 2016), pp. 1-6

12. QD Vu, KG Nguyen, M Juntti, in Proc. IEEE Global Commun. Conf. (GLOBECOM), Max-Min Fairness for Multicast Multigroup Multicell Transmission under Backhaul Constraints (IEEE, Washington, 2016), pp. 1-6

13. N Torabi, BS Ghahfarokhi, in Proc. 2014 4th Int. Conf. Comput. Knowl. Eng. (ICCKE), A TDMA-Based Channel Access Scheme for Achieving Fairness in InterVehicle Communications (IEEE, Mashhad, 2014), pp. 747-752

14. RL Batista, CF Silva, JM Silva, TF Maciel, FR Cavalcanti, in Proc. IEEE Wireless Commun. Netw. Conf. Workshops (WCNCW), What Happens with a Proportional Fair Cellular Scheduling when D2D Communications Underlay a Cellular Network (IEEE, Istanbul, 2014), pp. 260-265
15. ST Shah, J Gu, SF Hasan, MY Chung, SC-FDMA-based resource allocation and power control scheme for D2D communication using LTE-A uplink resource. EURASIP J. Wireless Commun. Netw. 2015, 137 (2015)

16. HH Wang, JC Chen, ZN Liu, in Proc. IEEE Global Commun. Conf. (GLOBECOM), Resource Allocation in Central-Controlled Device-to-Device Communications Networks (IEEE, Atlanta, 2013), pp. 4871-4876

17. F Kelly, Charging and rate control for elastic traffic. Euro. Trans. Telecommun. 8(1), 33-37 (1997)

18. 3rd Generation Partnership Project (3GPP) TS 36.300 V14.3.0, Technical Specification Group Radio Access Network; E-UTRA and E-UTRAN; Overall Description (2017)

19. DM Blough, G Resta, P Santi, in Proc. IEEE Conf. Comput. Commun. (INFOCOM), Interference-Aware Proportional Fairness for Multi-Rate Wireless Networks (IEEE, Toronto, 2014), pp. 2733-2741

20. TD Nguyen, Y Han, A proportional fairness algorithm with QoS provision in downlink OFDMA systems. IEEE Commun. Letters. 10(11), 760-762 (2006)

21. A Gjendemsjo, D Gesbert, GE Oien, SG Kiani, in Proc. International. Symposium on Modeling and Optimization in Mobile, Ad Hoc and Wireless Networks (WiOPT), Optimal Power Allocation and Scheduling for two-Cell Capacity Maximization (IEEE, Boston, 2006), pp. 1-6

22. A Gibbons, Algorithmic Graph Theory (Cambridge University Press, Cambridge, 1985), pp. 136-147

23. 3rd Generation Partnership Project (3GPP) TR 36.843 V12.0.1, Technical Specification Group Radio Access Network; Study on LTE Device to Device Proximity Services; Radio Aspects (2014)

\section{Submit your manuscript to a SpringerOpen ${ }^{\circ}$ journal and benefit from:}

- Convenient online submission

- Rigorous peer review

- Open access: articles freely available online

- High visibility within the field

- Retaining the copyright to your article

Submit your next manuscript at springeropen.com 